HURD - 9405

February, 1994.

\title{
ASYMPTOTICALLY FINITE THEORIES AND THE SCREENING OF COSMOLOGICAL CONSTANT BY QUANTUM EFFECTS
}

\author{
I. L. Shapirof \\ Tomsk State Pedagogical Institute, Tomsk, 634041, Russia \\ and \\ Department of Physics, Hiroshima University \\ Higashi - Hiroshima, Hiroshima, 724, Japan
}

\begin{abstract}
In the framework of the recently proposed asymptotically finite gauge models the cosmological constant is essentially weakened by quantum effects. The next (and more general) claim is that the coupling between quantum fields may suppress their contributions to the induced cosmological constant.
\end{abstract}

\footnotetext{
$\ddagger$ e-mail address: shapiro@fusion.sci.hiroshima-u.ac.jp
} 


\section{Introduction}

The cosmological constant problem is one of the more actual ones in a modern quantum field theory (see, for example, [1 - 7, 16 -21] and references there). Due to the observational data we know that the value of this constant is extraordinary small. At the same time the appearance of the cosmological constant is related with the vacuum energy which is generated in a variety of quantum field theory models at various energy scales (see [1] for an excellent review). Thus the known theories predict the nonzero value of the cosmological constant. In particular, the cosmological constant arise because of the symmetry breaking in a models of Grand Unification Theory (GUT) due to the nonzero vacuum expectation value for the Higgs bosons. The main purpose of this paper is to point out that for some class of GUT models there exist an effect of the renormalization group screening of the induced cosmological constant. The observable low-energy value of the cosmological constant is essentualy weakened. In fact the contributions to the cosmological constant arise within all the realistic quantum field theory models at all energy scales. One can suppose that the effect described below takes place (with a necessary modifications) at other energy scales and in the framework of another physical models.

\section{Asymptotically finite GUT's and screening of $\Lambda$}

In the present paper we shall restrict ourselves by consideration of the only one kind of GUT's with the special (asymptotically finite) kind of the renormalization group behaviour. Such models have been proposed in [8] (see also [9 - 11]). According to $[8,11]$ some asymptotically finite model corresponds to every finite one. All the beta - functions in asymptotical finite model tend to zero in both UV and IR limits. There is a lack of the zero - charge problem in both limits, and this fact is important for our purposes. The behaviour of finite and asymptotically finite models in curved space - time is of special interest. The action of general renormalizable gauge model in curved space - time can be written in the form (see [9] for the extensive review of renormalization in curved space time):

$$
S=\int d^{4} x \sqrt{-g}\left\{\mathcal{L}_{Y M}\left(A_{\mu}\right)+\mathcal{L}_{s c}(\Phi)+\mathcal{L}_{s p}(\Psi)+\mathcal{L}_{\text {int }}(A, \Phi, \Psi ; g, h, f)\right\}
$$

where $\mathcal{L}_{Y M}\left(A_{\mu}\right), \mathcal{L}_{s c}(\Phi), \mathcal{L}_{s p}(\Psi)$ are the Lagrangians of the Yang-Mills, scalar and spinor fields correspondingly and $\mathcal{L}_{\text {int }}(A, \Phi, \Psi ; g, h, f)$ is the interaction Lagrangian which depends on the set of gauge $g$, Yukawa $h$ and scalar (Higgs) $f$ couplings. The Lagrangian of

scalar field necessary contains the nonminimal term $\frac{1}{2} \xi R \Phi^{2}$. Moreover the action of the renormalizable theory contains also the vacuum terms [9] but we shall not deal with these 
terms here. In the framework of the asymptotically finite models there is no zero-charge problem in UV as well as in IR region. Thus one can use the perturbation theory in a consistent way in both regions. In external gravitational field the UV limit $t \rightarrow \infty$ corresponds to strong gravitational field with large values of curvature, and IR limit $t \rightarrow-\infty$ corresponds to weak gravitational field. The effective couplings in the matter sector have both UV and IR fixed points [8]. In patricular, when $t \rightarrow-\infty$ the effective constant $f(t)$ tends to some fixed value $f_{0}$. One can select some kind of asymptotycally finite theories which possess also the property of asymptotic conformal invariance [9,11,12]. The last means that in such theories the effective parameters of the nonminimal interaction $\xi(t)$ tend to the values, corresponding to the conformal theory. On the countrary, in IR limit these couplings grow indefinitely. It is important that in such models $\xi(t)$ grows exponentially [13,11], that is $\xi(t) \sim \exp \left(-A g^{2} t\right)$ where $A>0$ is some model - dependent constant, and $g$ is the fixed (nonzero) value of the gauge coupling.

Let us now pass to the cosmological constant problem. We start with the massless model and suppose that the scalar field mass arise as a result of dimensional transmutation within some phase transition. Then the values of the induced Newtonian $G$ and cosmological $\Lambda$ constants are defined by the values of $\left\langle\xi \Phi_{0}^{2}\right\rangle^{-1}$ and $\left\langle f \Phi_{0}^{4}\right\rangle$ where $\Phi_{0}$ corresponds to the minimum of the effective potential. The effective potential can be written as a series in loop parameter $\hbar$ as

$$
V_{e f f}=V+\sum_{n=1}^{\infty} \hbar^{n} V^{(n)}
$$

where $V=-\frac{1}{2} \xi R \Phi^{2}+f \Phi^{4}$ is classical potential of the theory (1). We consider the case of only one scalar field for simplicity. Since we are interesting in the low - energy consequences of the model under consideration, we have to explore the values of $\left\langle\xi \Phi_{0}^{2}\right\rangle^{-1}$ and $\left\langle f \Phi_{0}^{4}\right\rangle$ in the IR limit $t \rightarrow-\infty$. For these purposes we shall use the general solution of the renormalization group equation for effective potential in curved space-time (see, for example, [9]).

$$
V_{e f f}\left[e^{-2 t} g_{\alpha \beta}, \Phi, f, \xi, \mu\right]=e^{-4 t} V\left[g_{\alpha \beta}, \Phi(t), f(t), \xi(t), \mu\right]
$$

Here $\mu$ is dimensional parameter of renormalization. The effective coulings and fields satisfy the renormalization group equations of the form

$$
\begin{array}{cc}
\frac{d \Phi(t)}{d t}=\left(\gamma_{\Phi}-1\right) \Phi, & \Phi(0)=\Phi \\
\frac{d f(t)}{d t}=\beta_{f}, & f(0)=f \\
\frac{d \xi(t)}{d t}=\beta_{\xi}, & \xi(0)=\xi
\end{array}
$$


Generally speaking the exploration of the IR asymptotics of $G$ and $\Lambda$ needs the research of the corresponding limits of $V_{\text {eff }}$. At the same time one can obtain the general answer in a more simple way. Let us consider the zero order approximation for $V_{\text {eff }}$ taking the renormalization group improved classical potential $V$. Then we have to substitute the fields and couplings in the classical potential by the effective ones (4) according to (3). So we find

$$
\begin{aligned}
& G^{-1} \sim\left\langle\xi(t) \Phi_{0}^{2}(t)\right\rangle \\
& \frac{\Lambda}{G} \sim-\left\langle f(t) \Phi_{0}^{4}(t)\right\rangle
\end{aligned}
$$

Note that the beta - functions (4) are well - defined, unlike $\gamma_{\Phi}$ which is not. $\gamma_{\Phi}$ contains an essential arbitrariness, related with the dependence of the choice of gauge in the Yang - Mills sector and also with the arbitrariness in the parametrization of quantum fields. Therefore the only equation (4) for $\Phi$ is insufficient for the determination of the asymptotic of this effective charge. At the same time we can obviously find this asymptotic from ( $5 a)$. In fact, since $G(-\infty)$ have the finite classical value and $\xi(t) \sim \exp \left(-A g^{2} t\right)$ we find that $\Phi(t) \sim \exp \left(A g^{2} t\right)$. Now we take into account (5b). Since in the asymptotically finite models $\lambda(t) \rightarrow \lambda_{0}=$ const we find that in the IR limit $t \rightarrow-\infty$

$$
\Lambda \sim\left\langle f(t) \Phi_{0}^{4}(t)\right\rangle \sim \exp \left(2 A g^{2}\right) \rightarrow 0
$$

So we have found that in given approximation the cosmological constant decrease exponentially simultaneously with the decrease of the energy scale, and hence the observable value of $\Lambda$ will be essenially weakened.

In the above consideration we have used the renormalization group improved classical potential. In fact the higher loop contributions have the structure which differs from $V$ by some terms containing $\ln \Phi$. Since these terms have the softer IR behaviour in comparison with $\Phi^{4}$ or $\Phi^{2}$ one can obviously wait for just the same asymptotic behaviour of $V_{e f f}$ when the loop corrections are taken into account.

\section{Some attempt of numerical estimate}

Now we can try to make some numerical estimate of the effect considered above. Suppose that the asymptotically finite model describe the early Universe between some energy scales $\mu_{I R}$ and $\mu_{U V}$. Then from (4),(5b) and (6) we obtain the following relation for the ratio

$$
\frac{\Lambda_{I R}}{\Lambda_{U V}}=\left(\frac{\mu_{I R}}{\mu_{U V}}\right)^{2 A g^{2}}
$$


Hence the result of our estimate essentially depends on the choice of the model (that define $A$ ) as well as on the region of it's application. One can make, for instance, the following "optimistic" estimate. Let, for instance, the upper bound corresponds to Planck energies $\mu_{U V}=M_{p} \approx 10^{19} \mathrm{Gev}$ and the lower bound - to the energy of the relic radiation $\mu_{I R}=M_{r} \approx 10^{-12}$ Gev. The values of $A$ have been calculated in a number of papers (see, for example, $[13,12,11]$ and $[9]$ for finite and asymptotically finite models). Here we shall use the results of [11], where the bounds for $A$ are $5<A<50$. Taking the optimistic value $A=50$ we get

$$
\frac{\Lambda_{I R}}{\Lambda_{U V}} \approx 10^{-3100 g^{2}}
$$

Therefore if one suppose that $g \approx 10^{-1}$ then the value of $\Lambda$ is decreased on 31 order.

One can truly find above estimate as very naive. In fact we can not wait that some GUT model is valuable at more than 10 orders of the energy scale. Then we have to substitute 3100 in (8) by 1000 or even a less number. However there is another way to enlarge the power in (7). Generally speaking, $A$ is constant only in finite models, but not in asymptotically finite ones. If one consider the asymptotically finite theory at the middle (not UV or IR) character energies, the values of scalar and Yukawa couplings may differ from the fixed ones [8]. If one choose the large enough value for $f(0)$ then the coefficient $A$ will be larger (see [9] for the details of the renormalization group equation for $\xi$ ) and the considered effect will give more essential quantative improvements for the value of $\Lambda$ at low energies.

\section{Discussion}

We have considered the renormalization group mechanism of suppression of the cosmological constant. Some notes are in order. The described scheme doesn't need the fine tuning of the parameters of the theory. However if one want to deal with the asymptotically finite theories, the rigid resrictions on the multiplet composition takes place (see $[8,11]$ and references there for this point). One - loop models of this type have been proposed in [11] (see also [14] for some generalization concerning the finiteness in a massive sector). The direct use of the above scheme in higher loops needs the finiteness in the corresponding order of perturbation theory that is possible only in supersymmetric theories (see [15] and references there). However the renormalization groop screening of $\Lambda$ may take place in the non - finite models as well. In fact the more essential thing is a large enough value of the coupling $g$ and the choice of the model with large (and positive!) $A$ in (7). Therefore it is natural to suppose that the effective screening of $\Lambda$ is possible in an arbitrary kind of models with strong coupling. 
The discussed mechanism is based on the assumption that the necessary phase transition really takes place, and that the finite value of the gravitational constant is induced. Since we relate the values of induced constants $G$ and $\Lambda$ with the IR asymptotics of $\left\langle\xi \Phi_{0}^{2}\right\rangle$

and $\left\langle f \Phi_{0}^{4}\right\rangle$ it should be very interesting to explore the IR asymptotic behaviour of the corresponding composite operators, and so to get a more rigid prove for the screening of $\Lambda$.

\section{Acknowledgments}

Author is grateful to I.L.Buchbinder and I.V.Tyutin for helpful discussions. 


\section{References}

[1] Weinberg S., Rev.Mod.Phys., 61 (1989) 1

[2] Zeldovich Ya.B., JETPH Letters 6 (1967) 883

[3] Davies P.S.W., The Accidental Universe - Camdridge: Cambridge University Press. 1985

[4] Banks T., Nucl.Phys.B 249 (1985) 332

[5] Linde A.D., Phys. Lett. B 175 (1986) 395

[6] Abbot L., Phys.Lett. B 150 (1988) 427

[7] Peccei R.D., Sola J., Wetterich C.,Phys. Lett. B195(1987)183

[8] Odintsov S.D., Shapiro I.L., JETPH Letters 49 (1989) 125.

[9] Buchbinder S.D., Odintsov S.D., Shapiro I.L., Effective Action in Quantum Gravity.

- IOP Publishing, Bristol and Philadelphia .1992

[10] Konshtein S.E., Fradkin E.S., Kratkie soob. i pisma FIAN 12 (1986) 28.

[11] Shapiro I.L., Yagunov E.G., Int.J.Mod.Phys.A, 8 (1987) 1787.

[12] Buchbinder I.L., Shapiro I.L., Yagunov E.G., Sov.J.Nucl.Phys. 51 (1990) 249

[13] Buchbinder I.L., Odintsov S.D., Lihtzier I.M., Class.Quant.Grav. 6 (1989) 605

[14] Lucha W., Mozer M., Preprint Inst. Theor. Phys. Vienna (1993); Lucha W., Schoberl F., Preprint Inst. Theor. Phys. Vienna (1993)

[15] West P., Introduction to supersymmetry and supergravity.- World Scientific.Singapore-1986.

[16] Ellis J., Lahanas A.B., Nanopoulos D.V., Tamvakis K., Phys.Lett.B, 134 (1984) 429

[17] Ellis J., Tsamis N.C., Voloshin M.,Phys.Lett.B,194(1987)291

[18] Ford L.H., Phys.Rev.D,35 (1987) 2339

[19] Hawking S.W., Phys.Lett.B, 134 (1987) 403

[20] Antoniadis I., Mottola E., Phys.Rev.D, 45 (1992) 2013

[21] Antonoiadis I., Mazur P.O., Mottola E., Nucl.Phys.B388 (1992) 627 